\section{Concepções em disputa no uso da suplementação e/ou fortificação de micronutrientes na alimentação escolar para prevenção da anemia}

\section{Conceptions in dispute in the use of micronutrient supplementation and/or fortification in school programs for prevention of anemia}

\author{
Concepciones en disputa en el uso del uso \\ de suplementos y/o enriquecimiento de \\ micronutrientes en la alimentación escolar \\ para la prevención de la anemia
}

\author{
Patricia Camacho Dias 1 \\ Cyntia Gomes Teles 2 \\ Daniele Ferreira Mendonça 1 \\ Roseane Moreira Sampaio 1 \\ Patricia Henriques 1 \\ Daniele da Silva Bastos Soares 3 \\ Silvia Pereira 1 \\ Luciene Burlandy 1,4
}

doi: 10.1590/0102-311X00001321

\title{
Resumo
}

A anemia por deficiência de ferro afeta, mundialmente, diferentes populações e, no Brasil, é uma das principais carências nutricionais na infância. Diferentes estratégias são propostas por organismos internacionais e pelo Ministério da Saúde para sua prevenção. O objetivo do estudo foi analisar as ideias em disputa, presentes em documentos oficiais e narrativas dos profissionais da saúde e educação acerca do uso da suplementação e/ou fortificação nutricional como medida de prevenção da anemia no âmbito escolar. Baseou-se na análise de documentos governamentais e entrevistas semiestruturadas com profissionais atuantes em municípios que implementaram a estratégia de fortificação da alimentação escolar (NutriSUS) no Estado do Rio de Janeiro. As ideias disseminadas nos documentos governamentais ressaltam a eficiência da suplementação e da fortificação caseira para prevenção de anemia, com base em pesquisas científicas e se adequa aos objetivos das politicas públicas neles formalizados. As ideias presentes nas narrativas dos profissionais são, por vezes, críticas à necessidade desse tipo de intervenção e, por outras, favoráveis, indicando as controvérsias presentes no próprio processo de operacionalização local das politicas federais. Observaram-se tensões em torno dos conceitos de promoção, prevenção e tratamento por meio do NutriSUS, da dosagem e da forma de administração. As críticas à suplementação ressaltam as práticas alimentares saudáveis como estratégia preferencial. A medicalização em detrimento de ações de promoção da saúde pode tencionar a perspectiva pedagógica no contexto escolar e produzir ideias contraditórias sobre as melhores estratégias de promoção de alimentação saudável.

Política Pública de Saúde; Anemia; Alimentação Escolar;

Suplementos Nutricionais

\author{
Correspondência \\ P. C. Dias \\ Programa de Pós-graduação em Ciências da Nutrição, \\ Universidade Federal Fluminense. \\ Rua Mário Santos Braga 30, 4o andar, Niterói, RJ 24020-140, \\ Brasil. \\ diaspc2@gmail.com
1 Programa de Pós-graduação em Ciências da Nutrição, Universidade Federal Fluminense, Niterói, Brasil. 2 Universidade Federal Fluminense, Niterói, Brasil. 3 Faculdade de Nutrição Emília de Jesus Ferreiro, Universidade Federal Fluminense, Niterói, Brasil.
4 Programa de Estudos Pós-graduados em Política Social, Universidade Federal Fluminense, Niterói, Brasil.




\section{Introdução}

A anemia afeta, aproximadamente, $42,6 \%$ das crianças entre 6-59 meses no mundo 1 . No Brasil, atinge 18,9\% de crianças de 6-23 meses e 5,6\% de 24-59 meses com índices maiores, sobretudo, no Nordeste do país 2 . Seus fatores condicionantes incluem desde baixa ingestão de alimentos-fonte a baixa biodisponibilidade 3,4. As carências nutricionais culminam em diversos tipos de anemia e hipovitaminoses que aumentam a morbimortalidade infantil $4,5,6,7,8$, sendo que a anemia por deficiência de ferro na infância pode resultar em: retardo no desenvolvimento; redução de aprendizagem, perda cognitiva e agravamento de doenças infecciosas, especialmente em pré-escolares, pela demanda aumentada associada à baixa ingestão de alimentos-fonte na alimentação complementar e ao desmame precoce 5,9.

Diversas iniciativas já foram adotadas pelo governo brasileiro para a prevenção e o controle de anemia nessa faixa etária: a Estratégia Amamenta e Alimenta Brasil 10 estimula o aleitamento materno exclusivo até os seis meses e propõe um conjunto de diretrizes para a introdução gradual de alimentos para a primeira infância; o Programa Nacional de Suplementação de Ferro (PNSF) 11 disponibiliza suplementação isolada de ferro para crianças de 6-24 meses de idade, gestantes e mulheres pós-parto imediato; e a Resolução RDC no 344/2012, da Agência Nacional de Vigilância Sanitária (Anvisa) 12, define a fortificação com ferro e ácido fólico de farinhas de trigo e milho como estratégia preventiva para toda a população 4.

Em 2014, um novo esquema de suplementação de micronutrientes passou a ser adotado no Brasil, denominado NutriSUS (Estratégia de Fortificação da Alimentação Infantil com Micronutrientes em Pó), tendo como lócus de distribuição o ambiente escolar. O programa prevê a adição de um sachê composto por dosagens específicas de múltiplos micronutrientes à refeição pronta oferecida pelo Programa Nacional de Alimentação Escolar (PNAE) para pré-escolares e escolares 4,6,7,8. A estratégia passou a integrar o Programa Saúde na Escola (PSE), sendo incluída como uma ação de promoção de saúde e prevenção de doenças e agravos, visando garantir melhores condições para o pleno desenvolvimento, estimular hábitos alimentares saudáveis e enfrentar vulnerabilidades em saúde que possam comprometer o pleno desenvolvimento escolar 4,13. Todavia a suplementação em pó como estratégia de prevenção de anemia e promoção de alimentação saudável dentro do espaço escolar pode provocar tensão entre o NutriSUS e o PNAE quanto ao uso do ambiente escolar; à suplementação universalizada; à concepção de alimentação saudável e à concepção da alimentação escolar 14.

A convergência de distintos programas para o espaço escolar pode superpor objetivos e gerar constrangimentos, além das potenciais disputas de concepção. O PNAE objetiva contribuir para o crescimento e o desenvolvimento biopsicossocial, a aprendizagem, a formação de hábitos alimentares saudáveis; e o NutriSUS pretende potencializar o pleno desenvolvimento infantil, a prevenção e o controle da anemia. Elucidar e analisar as concepções presentes nos documentos oficiais e construídas pelos sujeitos pode contribuir para melhor entendimento dos caminhos e desafios desse tipo de estratégia, qual seja, a adição de suplemento em pó às refeições escolares. O objetivo do estudo foi analisar as ideias em disputa, presentes em documentos oficiais e narrativas dos profissionais da saúde e educação, acerca do uso da suplementação e/ou fortificação nutricional como medida de prevenção da anemia no âmbito escolar.

\section{Métodos}

\section{Desenho e referencial teórico}

Estudo qualitativo centrado na análise das ideias de diferentes segmentos de atores envolvidos com ações de suplementação nutricional, incluindo: (1) aquelas presentes no discurso governamental, acessado em documentos oficiais do governo federal; e (2) no discurso de profissionais que atuam em unidades de saúde, em escolas e instituições de Ensino Superior em municípios do Estado do Rio de Janeiro, Brasil, por meio de entrevistas.

O estudo foi realizado entre julho de 2018 e fevereiro de 2020 e baseou-se no referencial de análise de políticas públicas, com foco nas ideias em disputa sobre a estratégia de suplementação nutricional infantil no âmbito escolar como política de prevenção de anemia. Entende-se por ideia, 
a interpretação compartilhada pelos participantes e expressas pelos documentos oficiais analisados, ou seja, as estruturas de sentido apreendidas nas falas e textos que abarcam as convergências e divergências acerca do tema em questão 15,16. Os documentos oficiais foram concebidos como planos que norteiam a implementação, mas podem ser modificados pelo desenvolvimento da estratégia e interagem com as concepções construídas, em uma via de mão dupla 17,18,19. Considerou-se que a concepção dos sujeitos afeta e é afetada pela interação com o contexto e pela interpretação dos documentos orientadores $16,17,18,19$.

\section{Procedimentos metodológicos}

Os documentos analisados (Quadro 1) foram localizados nos endereços eletrônicos oficiais do Ministério da Saúde (https://www.gov.br/saude/pt-br) e do Ministério da Educação (https://www.gov.br/ $\mathrm{mec} / \mathrm{pt}-\mathrm{br})$.

As entrevistas foram realizadas presencialmente, gravadas, transcritas e analisadas. Os participantes compõem uma amostra não probabilística, selecionada por conveniência. Foram entrevistados 14 profissionais - oito da área da saúde e seis da educação -, incluindo dois docentes universitários que atuam na formação de nutricionistas em uma universidade pública, em razão da proximidade com o conteúdo teórico acerca do tema da suplementação nutricional. Todos pertenciam a municípios do Estado do Rio de Janeiro onde a estratégia estava sendo implementada. Para situar o setor e segmento do entrevistado, foram utilizados nas citações os seguintes códigos: PS para profissional da saúde, PE para profissional da educação e PD para profissional docente universitário, sendo todos seguidos do número de identificação da entrevista.

O material narrativo e os documentos oficiais foram analisados por meio de leitura exploratória e posterior análise de conteúdo com base nas seguintes dimensões pré-definidas: estratégias de prevenção da anemia; concepção de promoção de alimentação saudável e promoção da saúde que predomina na abordagem da anemia em escolas; universalização da estratégia de suplementação e escola como espaço para estratégias de suplementação nutricional.

O projeto foi aprovado pelo Comitê de Ética em Pesquisa da Faculdade de Medicina/Hospital Universitário Antônio Pedro da Universidade Federal Fluminense (CAAE no 98417718.5.0000.5243). Os entrevistados assinaram o termo de consentimento.

\section{Quadro 1}

Documentos públicos que abordam o combate à anemia infantil, selecionados para análise segundo o ano de publicação e órgão oficial responsável.

\begin{tabular}{|c|c|c|}
\hline TíTULO & FONTE & ANO \\
\hline Estratégia de Fortificação Caseira no Brasil 21 & Ministério da Saúde & 2012 \\
\hline $\begin{array}{l}\text { Diretriz: Uso de Formulações em Pó de Múltiplos Micronutrientes para Fortificação Caseira de } \\
\text { Alimentos Consumidos por Bebês e Crianças de } 6 \text { a } 23 \text { Meses de Vida } 22\end{array}$ & Organização Mundial da Saúde & 2013 \\
\hline Programa Nacional de Suplementação de Ferro - Manual de Condutas Gerais 11 & Ministério da Saúde & 2013 \\
\hline Suplemento Alimentar com Múltiplos Micronutrientes em Pó para Implantação do NutriSUS 8 & Ministério da Saúde & 2014 \\
\hline $\begin{array}{l}\text { NutriSUS - Estratégia de Fortificação da Alimentação Infantil com Micronutrientes (Vitaminas e } \\
\text { Minerais) em Pó: Manual Operacional } 4\end{array}$ & $\begin{array}{l}\text { Ministério da Saúde e } \\
\text { Ministério da Educação }\end{array}$ & 2015 \\
\hline $\begin{array}{l}\text { NutriSUS Guia de Evidências: Estratégia de Fortificação da Alimentação Infantil com Micronutrientes } \\
\text { (Vitaminas e Minerais) em Pó } 6\end{array}$ & Ministério da Saúde & 2015 \\
\hline $\begin{array}{l}\text { NutriSUS - Caderno de Orientações: Estratégia de Fortificação da alimentação infantil com } \\
\text { micronutrientes (Vitaminas e Minerais) em Pó } 7\end{array}$ & $\begin{array}{l}\text { Ministério da Saúde e } \\
\text { Ministério da Educação }\end{array}$ & 2015 \\
\hline
\end{tabular}

Fonte: elaboração própria. 


\section{Resultados e discussão}

Os resultados refletem a análise integrada das ideias acessadas por meio dos documentos selecionados e entrevistas realizadas. Consideraram-se as especificidades das fontes, uma vez que os documentos orientam e regulamentam as intervenções de prevenção e controle da anemia, e os depoimentos apresentam a concepção dos profissionais envolvidos com o processo local de implementação dessas ações. Em síntese, as ideias disseminadas nos documentos governamentais ressaltam a eficiência da suplementação e da fortificação caseira para prevenção de anemia, com base em pesquisas científicas realizadas em diversos países. Essa abordagem positiva da estratégia se adequa aos objetivos das políticas públicas neles formalizados. As ideias presentes nas narrativas dos profissionais são, por vezes, críticas à necessidade desse tipo de intervenção e, por outras, favoráveis, indicando as controvérsias presentes no próprio processo de operacionalização local das políticas federais. Esses diferentes tipos de discurso foram analisados de forma integrada a partir das dimensões sinalizadas anteriormente que configuraram eixos temáticos transversais abaixo relacionados, presentes nas narrativas dos diferentes segmentos de atores e que possibilitaram cotejar as distintas ideias que fomentam o debate público sobre suplementação.

\section{Conceitos em disputa - suplemento alimentar, fortificação caseira, suplemento medicamentoso}

As estratégias utilizadas para aumentar a ingestão de ferro pela população são classificadas em função da finalidade, composição, dose, público-alvo e local de implementação (Quadro 2). Observaram-se disputas em torno do conceito que caracteriza a estratégia NutriSUS.

Os documentos analisados se apropriam simultaneamente de duas definições como análogas: "suplementação" e "fortificação caseira". Destaca-se que o termo fortificação é utilizado na legislação brasileira 20 para descrever estratégias industriais de adição de nutrientes na produção de alimentos fortificados. No entanto, foi apropriado em alguns dos documentos 4,6,7,21,22 para caracterizar também a adição doméstica de nutrientes em pó em refeições, definindo-a como produto de fortificação caseira. A proposta do NutriSUS foi, portanto, inspirada em estratégias de fortificação no ambiente doméstico, redefinidas para o espaço escolar.

Nas entrevistas, identificou-se que os distintos termos foram apropriados referindo-se a estratégias diferentes, como observado na seguinte fala: "acho que seria interessante a suplementação, mas a escola é muito completa, (...), na minha visão como nutricionista, não teria uma necessidade de introduzir um pó, poderia introduzir uma fortificação talvez até do próprio alimento" (PE1). Outro depoente afirma: "não é muito bem apenas um suplemento ali, é um polivitaminico, um polimineral" (PD1).

Nessa narrativa, parece haver uma compreensão do NutriSUS como um medicamento. É válido ressaltar que uma parcela dos profissionais, tanto da saúde quanto da educação, desconhecia o NutriSUS, mas ainda assim, em sua maioria, o apresentavam como estratégia de suplementação.

\section{Estratégias de prevenção de anemia}

Os documentos convergem ao definir o uso do suplemento como medida preventiva e, também, o definem como estratégia de promoção de saúde e de alimentação saudável 6 . As entrevistas realizadas, entretanto, mostram opiniões diversas a respeito de estratégias para a prevenção de anemia, não trazendo, contudo, divergências marcantes de acordo com a área de atuação do profissional, ou seja, os profissionais de um mesmo segmento não necessariamente concordam entre si ou elencam, integralmente, as mesmas estratégias preventivas (Quadro 3).

A alimentação saudável e a oferta de alimentos fonte de ferro foram as estratégias destacadas de modo unânime pelos diferentes setores entrevistados, incluindo a consolidação de políticas públicas que promovam esse acesso. A educação alimentar e nutricional foi a quarta estratégia mais citada, seguida do uso de suplementos no âmbito da saúde (PNSF) e no âmbito da educação (NutriSUS), além da fortificação de farinhas prevista em lei. 
Quadro 2

Definições dos diferentes tipos de suplemento de acordo com a legislação vigente ou documento de referência.

\begin{tabular}{|c|c|c|}
\hline TIPO & DEFINIÇÃO & $\begin{array}{c}\text { DOCUMENTOS OFICIAIS E } \\
\text { LEGISLAÇÃO }\end{array}$ \\
\hline $\begin{array}{l}\text { Suplemento } \\
\text { medicamentoso }\end{array}$ & $\begin{array}{l}\text { Produto à base de vitaminas e minerais com finalidade terapêutica ou } \\
\text { medicamentosa, ou seja, prevenção ou tratamento de doenças. Os medicamentos } \\
\text { à base de vitaminas e minerais são definidos como produtos à base de vitaminas } \\
\text { ou minerais com finalidade profilática, curativa ou paliativa, cujos esquemas } \\
\text { posológicos diários situam-se acima de } 100 \% \text { da IDR (pelo menos um dos } \\
\text { componentes). Podem ser classificados em medicamentos sem prescrição médica. }\end{array}$ & Portaria MS/SVS no 40/199859 \\
\hline Suplemento alimentar & $\begin{array}{l}\text { Alimentos que servem para complementar com vitaminas e/ou minerais a dieta } \\
\text { diária de uma população saudável, em casos em que sua ingestão, a partir da } \\
\text { alimentação, seja insuficiente ou quando a dieta requer suplementação. Sua } \\
\text { composição deve ser no mínimo } 25 \% \text { e no máximo } 100 \% \text { da IDR de vitaminas e/ou } \\
\text { minerais indicada pelo fabricante. Não podem substituir os alimentos nem serem } \\
\text { considerados como dieta exclusiva e não incluem produtos indicados para prevenir, } \\
\text { aliviar, tratar uma enfermidade ou alteração do estado fisiológico. }\end{array}$ & $\begin{array}{l}\text { Portaria MS/SVS no 32/1998 } 34 \text { e } \\
\text { Resolução RDC no 269/2005 } 35\end{array}$ \\
\hline $\begin{array}{l}\text { Produto de fortificação } \\
\text { caseira }\end{array}$ & $\begin{array}{l}\text { (1) Mistura de micronutrientes em pó para formar qualquer alimento semissólido. A } \\
\text { mistura é fornecida em sachês de porção individual e o conteúdo é salpicado sobre } \\
\text { o alimento antes do consumo. A intervenção pode ser feita em casa ou em qualquer } \\
\text { outro lugar onde as refeições são consumidas. } \\
\text { (2) Uso de múltiplos micronutrientes que visa potencializar o pleno } \\
\text { desenvolvimento infantil e a prevenção e controle da deficiência de vitaminas e } \\
\text { minerais, particularmente da anemia e deficiência de ferro mediante a adição direta } \\
\text { de múltiplos nutrientes em pó aos alimentos. } \\
\text { (3) Produto fornecido em sachês individuais de múltiplos micronutrientes na } \\
\text { forma de pó para adicionar ao alimento pronto, sem alterar as características } \\
\text { organolépticas da comida. }\end{array}$ & $\begin{array}{l}\text { Não possui legislação vigente. } \\
\text { (1) Estratégia de Fortificação } \\
\text { Caseira no Brasil (2012) 21; (2) } \\
\text { Diretriz: Uso de Formulações em Pó } \\
\text { de Múltiplos Micronutrientes para } \\
\text { Fortificação Caseira de Alimentos } \\
\text { Consumidos por Bebês e Crianças } \\
\text { de 6-23 Meses de Vida (2013) 22; } \\
\text { (3) NutriSUS Guia de Evidências: } \\
\text { Estratégia de Fortificação } \\
\text { da Alimentação Infantil com } \\
\text { Micronutrientes (Vitaminas e } \\
\text { Minerais) em Pó (2015) } 6 \text {. }\end{array}$ \\
\hline $\begin{array}{l}\text { Alimento fortificado, } \\
\text { enriquecido ou } \\
\text { adicionado de outros } \\
\text { nutrientes }\end{array}$ & $\begin{array}{l}\text { Todo alimento ao qual for adicionado um ou mais nutrientes essenciais } \\
\text { contidos naturalmente ou não no alimento, com objetivo de reforçar seu valor } \\
\text { nutritivo e/ou prevenir ou corrigir deficiência(s) demonstrada(s) em um ou mais } \\
\text { nutrientes, na alimentação da população ou em grupos específicos. A adição de } \\
\text { nutrientes em alimentos fortificados não deve resultar em ingestão excessiva ou } \\
\text { insignificante do nutriente adicionado, considerando as quantidades derivadas } \\
\text { de outros alimentos da dieta e as necessidades do consumidor. Deve considerar } \\
\text { a possibilidade de ocorrência de interação negativa com nutrientes ou outros } \\
\text { componentes do alimento. O nutriente deve ser biodisponível ou seguro, e a adição } \\
\text { não deve alcançar níveis terapêuticos no alimento. Esses alimentos podem ter } \\
\text { fins comerciais ou serem voltados para programas institucionais. Para alimentos } \\
\text { fortificados ou enriquecidos, é permitida a fortificação/enriquecimento, desde } \\
\text { que apresentem no mínimo 15\% da IDR de vitaminas e minerais em 100mL } \\
\text { ou, no mínimo, 30\% da IDR de vitaminas e minerais em 100g de produto. Nos } \\
\text { alimentos enriquecidos/fortificados para programas institucionais, é permitido o } \\
\text { enriquecimento/fortificação sempre que houver justificativa de ordem nutricional } \\
\text { reconhecida por órgão competente comprovando níveis baixos de ingestão por } \\
\text { estudos epidemiológicos, que o alimento usado como veículo seja consumido } \\
\text { significativamente pela população vulnerável, que a adição seja compatível com o } \\
\text { déficit da população afetada. }\end{array}$ & Portaria MS/SVS no 31/1998 20 \\
\hline
\end{tabular}

IDR: ingestão diária recomendada.

Fonte: elaboração própria. 
Quadro 3

Argumentos apresentados nos documentos e entrevistas a respeito da suplementação como estratégia de prevenção de saúde em escola.

\begin{tabular}{|c|c|c|c|c|}
\hline \multirow[t]{2}{*}{ ARGUMENTOS } & \multicolumn{3}{|c|}{ DEPOENTES } & \multirow[t]{2}{*}{ DOCUMENTOS } \\
\hline & DOCÊNCIA & SAÚDE & EDUCAÇÃO & \\
\hline \multicolumn{5}{|l|}{ Favoráveis } \\
\hline $\begin{array}{l}\text { Grande captação em decorrência do tempo que as crianças permanecem } \\
\text { na escola }\end{array}$ & & $x$ & $x$ & $x$ \\
\hline Alta prevalência de anemia na faixa etária específica & & & & $x$ \\
\hline Escola como ambiente que permite controle da alimentação dos alunos & & $X$ & $\mathrm{X}$ & \\
\hline $\begin{array}{l}\text { Escola como ambiente que permite controle da administração e descarte } \\
\text { correto do sachê }\end{array}$ & & & $x$ & $x$ \\
\hline Fragilidade social vivida por maioria dos estudantes & & & $\mathrm{X}$ & \\
\hline Cardápio escolar pouco adequado & & $\mathrm{X}$ & & $\mathrm{X}$ \\
\hline $\begin{array}{l}\text { Os alunos recebem na escola o acompanhamento de nutricionista ou } \\
\text { profissional educador }\end{array}$ & & $x$ & $x$ & $x$ \\
\hline $\begin{array}{l}\text { Essa faixa etária está formando a seletividade de paladar e o suplemento } \\
\text { permitiria driblar baixa aceitabilidade }\end{array}$ & & & $x$ & $x$ \\
\hline \multicolumn{5}{|l|}{ Contrários } \\
\hline $\begin{array}{l}\text { A escola deve promover a educação alimentar e nutricional com alimentos, } \\
\text { formando hábitos saudáveis. A suplementação não promove educação }\end{array}$ & $x$ & $\mathrm{x}$ & $x$ & \\
\hline $\begin{array}{l}\text { A escola deve priorizar qualidade da merenda escolar, e não } \\
\text { suplementação }\end{array}$ & $x$ & $x$ & & \\
\hline A suplementação deve ser acompanhada de qualidade no cardápio escolar & & & $\mathrm{X}$ & \\
\hline $\begin{array}{l}\text { Não existe profissional suficiente para controle da estratégia nas } \\
\text { instituições de ensino }\end{array}$ & $\mathrm{X}$ & & $\mathrm{X}$ & \\
\hline $\begin{array}{l}\text { A escola não tem aparato técnico e tecnológico para fazer triagem dos } \\
\text { alunos e verificar os que de fato precisam de suplementação }\end{array}$ & $x$ & $\mathrm{X}$ & $\mathrm{X}$ & \\
\hline
\end{tabular}

Fonte: elaboração própria.

Os docentes universitários apresentaram posicionamento crítico e desfavorável ao uso de suplementos para essa faixa etária e destacaram que a educação alimentar e nutricional e a alimentação escolar sem adições devem ser reforçadas para favorecer a formação de hábitos que podem perpetuar por toda a vida adulta.

Houve discordância entre os participantes acerca da universalização da adição do pó as refeições escolares como estratégia. Os profissionais da saúde e educação em cargos de gestão possuíam maior afinidade com a estratégia de suplementação. Em contrapartida, os profissionais que atuam nas escolas pareciam ter algumas ressalvas, trazendo questões como a importância e o papel central da educação e da qualidade do cardápio escolar. Por fim, o segmento da docência posicionou-se contra o NutriSUS e a suplementação universalizada como estratégia preventiva.

Os profissionais com maior familiaridade com o NutriSUS se posicionaram favoráveis, mesmo que entre eles houvesse opinião desfavorável à suplementação em escolas sem avaliação prévia, como ocorre na estratégia. As principais críticas ao programa apontaram a falta de avaliação bioquímica antes e depois da administração do sachê para averiguar a real necessidade de suplementação e evidenciar a efetividade ou não da estratégia. A ausência de cunho pedagógico também foi mencionada como crítica ao programa, destacando que a educação alimentar e nutricional não é elencada em documento oficial 6 como um dos objetivos da intervenção. 


\section{Promoção, prevenção ou tratamento medicamentoso}

Em relação à dose de micronutrientes no sachê bem como à biodisponibilidade, observaram-se divergências de discurso. Alguns entrevistados relataram não concordar sobre a dosagem escolhida porque determina uma mesma quantidade para diferentes faixas etárias que possuem diferentes recomendações dietéticas de ingestão.

Portanto, houve divergência entre alguns entrevistados se a quantidade administrada se tratava de dose de prevenção ou de tratamento. Nos documentos analisados 4,6,7, a estratégia é definida como de prevenção, tendo como argumento a dosagem sugerida ${ }^{7}$. A fortificação caseira é indicada em documento 6 como uma alternativa a intervenções medicamentosas. A justificativa para esta diferenciação de conceitos é que a fortificação caseira usa o alimento como base, sendo incorporada às práticas alimentares conforme consta nos documentos analisados 6 .

Segundo os documentos do Ministério da Saúde 4,7, a composição do sachê do NutriSUS adotado no Brasil foi definida com base na recomendação descrita no documento Programmatic Guidance Brief on Use of Micronutrient Powders (MNP) for Home Fortification 23, que estabeleceu as respectivas quantidades com a intenção de assegurar a prevenção de deficiência dos micronutrientes descritos para crianças de 6-59 meses de idade, faixa considerada de risco. Este documento aponta a escola como possível espaço para o uso do suplemento, principalmente naquelas escolas cujo cardápio fornece micronutrientes de forma limitada 23.

Nos documentos 4,6,7, identificam-se argumentos balizados na composição dos saches com múltiplos micronutrientes, destacando que: são mais eficientes ou tão eficientes quanto a suplementação de ferro isolada para prevenção de anemia; permitem maior aceitabilidade por não gerar alterações gastrointestinais, escurecimento dentário e vômitos, como observado na suplementação isolada de ferro; não alteram cor, sabor e textura dos alimentos e aumentam a biodisponibilidade 4,6,7. Esse argumento se contrapõe com a seguinte fala de um dos entrevistados: "dentro desse programa, pelo menos o objetivo principal é combater a carência de ferro e foca na anemia, mas o pó tem vários outros nutrientes, que acabam sendo... em termos de biodisponibilidade, competem uns nutrientes com os outros" (PD1).

Ressalta-se que, em documento oficial 4, o NutriSUS é definido como medida de promoção da saúde e de promoção de alimentação saudável. Assim, cabe a reflexão das implicações em considerá-lo como "medicamento", especialmente em um contexto em que a oferta da alimentação escolar é orientada pelo PNAE 24,25 que tem a promoção de alimentação saudável e a segurança alimentar e nutricional como referências 26. A implantação do NutriSUS pode, assim, favorecer a construção de novas narrativas e concepções acerca do próprio PNAE, de modo a considerá-lo insuficiente para garantir uma boa alimentação escolar. Uma vez que a execução de uma política pública compreende a interpretação das diretrizes governamentais 16, não se pode desconsiderar que tanto as concepções constroem as práticas quanto estas forjam novas concepções 18,19 .

A promoção de saúde pode ser compreendida com base em diferentes abordagens e, por vezes, a prevenção de doenças ganha maior ênfase do que uma perspectiva positiva de promoção da saúde 27,28,29,30. No caso do NutriSUS, evidencia-se a predominância de uma abordagem preventiva baseada no modelo biomédico, pois não prevê ações que visem mudanças ambientais, nas práticas ou nos modos de vida 27,31 .

Os documentos oficiais apresentam uma narrativa que tenta descaracterizar o NutriSUS como medicamento. Assim, o sachê de micronutrientes é definido como dose de prevenção, mencionado como opção à intervenção medicamentosa ${ }^{6}$. Contudo é listado na Relação Nacional de Medicamentos Essenciais (Rename) de 2020 32, no âmbito do Sistema Único de Saúde (SUS), e no relatório da Comissão Nacional de Incorporação de Tecnologias no SUS (CONITEC) 8, em que se descreve que a produção nacional desse medicamento é fruto de uma parceria para o desenvolvimento produtivo (PDP) mediante termo assinado em 2013 entre o Ministério da Saúde e o Laboratório Farmacêutico da Marinha do Brasil 33. De acordo com o documento, a Anvisa publicou, em 2018, nova resolução que classificava o NutriSUS como suplemento alimentar, e não como suplemento medicamentoso, para facilitar a aquisição do produto em 2019 33. Percebe-se que o enquadramento do NutriSUS como suplemento alimentar foi demandado após o início de execução da estratégia, que se deu a partir de 2014, sugerindo uma disputa na concepção nos documentos orientadores 4,6,7. 
No entanto, mesmo enquadrado como suplemento alimentar, seus propósitos e desenho de oferta não se alinham ao previsto na legislação sanitária, segundo a qual um suplemento alimentar precisa atingir entre $25 \%$ e $100 \%$ da ingestão diária recomendada (IDR) do grupo-alvo, e não pode ser vinculado com qualquer expressão que se refira ao seu uso como de prevenção ou alteração do estado fisiológico 34 , objetivos tais que o NutriSUS se propõe a cumprir. No que se refere à dosagem que compõe o sachê, as quantidades foram determinadas a partir de um documento específico 23 que ultrapassa o valor limite de $100 \%$ da IDR para ferro, zinco, cobre e ácido fólico em crianças de 1-3 anos, como estipulado pela mesma Portaria no 32/1998 35,36,37. O NutriSUS dispõe da mesma quantidade de micronutrientes para diferentes faixas etárias e não considera particularidades como peso e tipo de parto, prerrogativas levadas em conta em outras recomendações como da Organização das Nações Unidas para a Alimentação e a Agricultura (FAO, 2001) 37, do PNSF (2013) 11 e da Sociedade Brasileira de Pediatria (2012) 38.

Ao que parece, o NutriSUS não se adequa em nenhuma das definições já existentes e previstas em legislação, o que pode justificar o uso do termo "fortificação caseira" nos documentos analisados. Este termo foi adaptado de um insumo já difundido no Canadá, desde 1996, o Sprinkles, cuja intenção era ser vendido como sachê infantil de micronutrientes em pó para prevenção de anemia 6,39. Essa experiência vem sendo realizada em diversos países considerados subdesenvolvidos ou em desenvolvimento $40,41,42,43$.

Os documentos nacionais 4,6,7 se apropriam de resultados de estudos realizados em outros países para corroborar a eficiência e eficácia do suplemento. Nenhum dos estudos apresentados foi realizado no Brasil e poucos se referem à escola como potencial espaço para suplementação, tendo sido, em maioria, implementado em outros espaços como unidade de saúde ou residências 23,44 . Portanto o desenho da estratégia pode não ecoar particularidades do perfil social e alimentar das crianças, e ao contexto da política educacional e alimentar brasileira, que oferece um panorama diferenciado dos demais países.

\section{Escola como espaço para estratégias de suplementação nutricional}

Identificaram-se diversos argumentos nos documentos e entrevistas para justificar ou contestar a suplementação em ambiente escolar. No Quadro 4, observa-se a divergência entre os participantes quanto à adequação da escola como ambiente para estratégia de suplementação, sem distinção considerável entre os segmentos da saúde e educação. Apenas o segmento de docentes universitários foi contrário a esse tipo de estratégia.

Os principais argumentos favoráveis foram fundamentados na condição social dos escolares. Segundo alguns participantes, a escola seria o ambiente que garantiria o acesso e controle da qualidade da alimentação deste público, bem como da administração deste suplemento: "acho adequado a suplementação, até porque, muitas vezes, são as únicas refeições que eles têm são ali [na escola], então vai pra casa, come essas coisas industrializadas, esses alimentos industrializados, e às vezes também se dilui entre os irmãos" (PE3). E seu uso também se justificaria pelos hábitos alimentares ruins dessas crianças e pelo cardápio inadequado em escolas: "se você pegar mesmo todo mundo, com a dificuldade que está, ninguém tem uma alimentação balanceada (...), nem as próprias creches oferecem uma alimentação balanceada, é rica em carboidrato" (PS3). Como visto anteriormente, uma possível inadequação do cardápio aparece nos documentos oficiais do Ministério da Saúde e na narrativa dos participantes como justificativa para o NutriSUS. No entanto, as diretrizes alimentares do PNAE balizam cardápios adequados, o que não significa que em alguns contextos locais identifique-se algum tipo de inadequação. É preocupante que essa ideia de inadequação, ou insuficiência, esteja presente nessas narrativas, inclusive oficiais, e sinalize para soluções complementares a alimentação escolar, e não para medidas de ajustes onde forem necessárias. A disseminação desse tipo de ideia pode afetar as concepções sobre o próprio PNAE.

Alguns participantes questionaram a necessidade real de suplementação e apontaram a necessidade de triagem com avaliações bioquímicas que comprovasse a carência nutricional. Mesmo os favoráveis à suplementação na escola destacaram seu papel educativo, indicando que poderiam coexistir ambas as ações dentro do mesmo espaço.

Alguns documentos 4,7 reforçam que o ambiente escolar permite a captação dos alunos por meio de controle de presença, atuação do nutricionista e treinamento adequado de merendeiras, possibili- 
Quadro 4

Estratégias de prevenção de anemia infantil de acordo com profissionais entrevistados e documentos analisados.

\begin{tabular}{|c|c|c|c|c|}
\hline \multirow[t]{2}{*}{ CATEGORIAS } & \multicolumn{3}{|c|}{ DEPOENTES } & \multirow[t]{2}{*}{ DOCUMENTOS } \\
\hline & DOCÊNCIA & SAÚDE & EDUCAÇÃO & \\
\hline Fortificação caseira & & $\mathrm{X}$ & $\mathrm{X}$ & $\mathrm{X}$ \\
\hline Suplementação de ferro & & $\mathrm{X}$ & $\mathrm{X}$ & $\mathrm{X}$ \\
\hline Nutrisus & & & $x$ & $x$ \\
\hline Alimentação saudável & $\mathrm{X}$ & $\mathrm{x}$ & $x$ & $x$ \\
\hline Política pública que garanta acesso à alimentação saudável & $\mathrm{x}$ & $\mathrm{x}$ & $x$ & \\
\hline Alimentação com alimentos fonte de ferro & $\mathrm{X}$ & $\mathrm{X}$ & $x$ & $x$ \\
\hline Incentivo ao aleitamento materno exclusivo até 6 meses & $\mathrm{x}$ & & & $\mathrm{X}$ \\
\hline Acompanhamento pré-natal & $\mathrm{X}$ & $\mathrm{X}$ & & \\
\hline Educação nutricional & $\mathrm{X}$ & & $x$ & \\
\hline $\begin{array}{l}\text { Adequação do cardápio escolar ao proposto pelo PNAE/cardápios escolares } \\
\text { balanceados }\end{array}$ & & $\mathrm{X}$ & $x$ & \\
\hline Controle de parasitoses & & & & $\mathrm{x}$ \\
\hline
\end{tabular}

PNAE: Programa Nacional de Alimentação Escolar.

Fonte: elaboração própria.

tando a administração adequada do sachê de micronutrientes. A escola é citada como ambiente onde se estabelece rotina e desenvolve hábitos, por isso a implementação deveria ocorrer nesse espaço. Além disso, a suplementação foi defendida para essa faixa etária em decorrência da alta prevalência de anemia em consequência do aumento da carência nutricional, característico deste grupo, que também está exposto a práticas alimentares mais industrializadas e pouco nutritivas. Tais argumentos também foram reproduzidos por alguns entrevistados.

Os tipos de fortificação apresentados em grande parte dos artigos científicos 45,46,47,48, usados como defesa da eficácia da estratégia de suplementação, se caracterizavam, na verdade, como fortificação em nível industrial, fortificação em ambiente doméstico ou suplementação medicamentosa em rede de saúde. A adoção da escola como espaço para suplementação é ainda tema pouco explorado e sem estudos em território brasileiro. Há que se considerar as dificuldades na implementação de estratégias de suplementação em unidades de saúde e no espaço doméstico 14.

Seu desenho tem implicações para a dimensão operacional; para as concepções acerca da promoção de alimentação saudável no âmbito do NutriSUS e do PNAE; para a medicalização da alimentação e para a leitura pedagógica da suplementação. Além disso, enquanto a fortificação caseira ocorre sob o controle de um responsável, na escola, um único profissional é responsável por várias crianças, com impacto na operacionalização da estratégia, conforme destacado por entrevistados.

A estratégia parece ainda divergir de outros documentos de referência do Ministério da Saúde para promoção da saúde e alimentação saudável 49,50,51,52,53. Um dos documentos do NutriSUS 6 apresenta sugestões de cardápios de acordo com as orientações do Guia Alimentar para Crianças Brasileiras Menores de 2 Anos 53, com o objetivo de comparar o aporte total de ferro do cardápio com as recomendações diárias sugeridas pelo Instituto de Medicina dos Estados Unidos ${ }^{54}$ para crianças de até 12 meses, demonstrando a insuficiência do esquema alimentar proposto. Essa narrativa é utilizada como argumento para comprovar a necessidade de suplementação. Destaca-se que a recente revisão do mesmo guia 53 afirma que a prevenção de anemia por deficiência de ferro deve privilegiar o aleitamento materno e dieta com alimentos fontes do nutriente, sendo o uso de suplemento condicionado à prescrição de profissional de saúde.

Os guias alimentares para a população brasileira adulta 49,52 apontam que os suplementos nutricionais de vitaminas e minerais não são necessários se as orientações ali propostas forem seguidas indicando o uso apenas individual e após orientação de um médico ou nutricionista. Segundo esses 
documentos, o consumo de frutas, legumes e verduras confere uma proteção contra doenças que não se observa com uso isolado de nutrientes.

O documento Escola Promotoras de Saúde: Experiências no Brasil 50 também apresenta posição contrária à medicalização em escolas e procura desestimular seu uso nesses ambientes. Apesar de não fazer referência específica à deficiência de ferro, este documento trata da escola como local para cuidados progressivos com a saúde que contribuam para consolidação do SUS, se referindo a ações preventivas, educativas e emergenciais conjuntas de profissionais da educação e saúde, e que contribuam para a desmedicalização em escolas.

A adição de um pó às refeições escolares, aliada às práticas alimentares inadequadas, parece reforçar a ideia de insuficiência da alimentação escolar, como dito anteriormente. Destaca-se que o PNAE é o programa mais importante para a garantia da alimentação adequada e saudável no contexto escolar. Ao longo de sua trajetória, aprimorou, de forma sistemática, as diretrizes alimentares, estabelecendo parâmetros mínimos para a oferta de frutas e hortaliças, restringindo alimentos considerados não saudáveis, garantindo aporte de nutrientes, conforme recomendações, e proibindo determinados alimentos considerados inadequados para o consumo de crianças e adolescentes 24,25,55.

O uso do sachê implica em alterações no cardápio escolar para que a refeição seja apropriada para veicular o complexo de micronutrientes, exigindo condições específicas de consistência e temperatura. Além disso, demanda mudanças na dinâmica do preparo e distribuição das refeições, aspectos pouco valorizados nos documentos oficiais 4,7 . A disputa de concepções e princípios entre programas que convergem para o mesmo espaço institucional pode repercutir negativamente em ambos 14,56,57 e produzir resultados não esperados e/ou indesejáveis, tais como resistência de profissionais, concepções de alimentação adequada e saudável contraditórias para escolares e responsáveis, e mudança na lógica dos programas.

Estratégias intersetoriais, como é o caso tratado neste estudo, demandam articulações e construção de consensos entre os diferentes setores, cada um com suas perspectivas de atuação e interesses 14,56,57. Nesse sentido, há que se destacar os interesses da indústria farmacêutica que contribuem para medicalizar a promoção da saúde ao sugerir que os nutrientes contidos nos suplementos são mais eficazes do que os dos alimentos 58 . A suplementação preventiva em instituições escolares coloca luz sobre distintas abordagens da promoção de alimentação saudável que podem disputar espaço na construção de narrativas e práticas.

\section{Conclusão}

É consensual que existe a necessidade de ações voltadas para prevenção de anemia, bem como de promoção de saúde e de alimentação adequada e saudável. Foram observadas tensões em torno dos conceitos de promoção, prevenção e tratamento a partir do NutriSUS. As diferentes interpretações acerca da dosagem e da forma de administração dificultam o entendimento da estratégia quanto aos seus objetivos.

Um dos elementos importantes para compreender os consensos e dissensos em torno da utilização da suplementação ou fortificação como solução para deficiências alimentares é considerar que essas práticas vêm se consolidando como tendências transnacionais no contexto dos sistemas alimentares agroindustriais, influenciando as concepções de diferentes segmentos de atores. As críticas caminham na contramão dessas tendências e na direção de fortalecer práticas alimentares adequadas e saudáveis como forma de prevenir e controlar as deficiências alimentares. A adoção de práticas medicalizantes em detrimento de ações de promoção da saúde pode tencionar a perspectiva pedagógica mais ampla no contexto escolar e produzir concepções e ideias contraditórias sobre as melhores estratégias de promoção de alimentação saudável.

A escassez de estudos sobre suplementação infantil profilática em ambiente escolar, em especial em território brasileiro, corrobora para dificuldade de sustentar estratégias que podem implicar em leituras negativas de políticas públicas já existentes, bem como dos benefícios da alimentação balanceada, diversificada e segura. Foram identificadas tensões em torno da escola como espaço para a suplementação. Os argumentos que justificam a suplementação ficaram em torno da capilaridade da escola, da vulnerabilidade social dos escolares, das práticas alimentares inadequadas e do potencial 
controle operacional do programa. As posições contrárias focaram em argumentos em torno do papel educativo da escola, da crítica à universalização da suplementação e do papel da escola na promoção da saúde. Destaca-se que o estudo não avançou na análise da operacionalização do NutriSUS e a avaliação dos seus efeitos sobre o panorama da anemia nos diferentes cenários deve integrar uma agenda de pesquisa necessária nesse campo.

\section{Colaboradores}

P. C. Dias, D. F. Mendonça e R. M. Sampaio contribuíram com a concepção do estudo, análise e interpretação dos dados, redação e revisão crítica. C. G. Teles contribuiu com a coleta, análise e interpretação dos dados, redação do artigo e revisão crítica. P. Henriques, D. S. B. Soares, S. Pereira e L. Burlandy contribuíram com a análise e interpretação dos dados, redação do artigo e revisão crítica. Todas as autoras aprovaram a versão final a ser publicada.

\section{Informações adicionais}

ORCID: Patricia Camacho Dias (0000-0002-06748832); Cyntia Gomes Teles (0000-0001-8836-8368); Daniele Ferreira Mendonça (0000-0001-71228270); Roseane Moreira Sampaio (0000-00020850-7143); Patricia Henriques (0000-0001-81540962); Daniele da Silva Bastos Soares (0000-00015196-9055); Silvia Pereira (0000-0003-1538-4097); Luciene Burlandy (0000-0003-0875-6374).

\section{Agradecimentos}

Este trabalho foi realizado com apoio da Coordenação de Aperfeiçoamento de Pessoal de Nível Superior (CAPES) e da Fundação Carlos Chagas Filho de Amparo à Pesquisa do Estado do Rio de Janeiro (FAPERJ). L. Burlandy recebe bolsa PQ do Conselho Nacional de Desenvolvimento Científico e Tecnológico (CNPq; processo \#313714/2020-7).

\section{Referências}

1. World Health Organization. The global prevalence of anemia in 2011. Geneva: World Health Organization; 2015.

2. Universidade Federal do Rio de Janeiro. Estudo Nacional de Alimentação e Nutrição Infantil - ENANI-2019: Resultados preliminares - prevalência de anemia e deficiência de vitamina A entre crianças brasileiras de 6 a 59 meses. Rio de Janeiro: Universidade Federal do Rio de Janeiro; 2020.

3. World Health Organization. Guideline: use of multiple micronutrient powders for pointof-use fortification of foods consumed by infants and young children aged 6-23 months and children aged 2-12 years. Geneva: World Health Organization; 2016.

4. Ministério da Saúde. NutriSUS - estratégia de fortificação da alimentação infantil com micronutrientes (vitaminas e minerais) em pó: manual operacional. Brasília: Ministério da Saúde/Ministério da Educação; 2015.

5. World Health Organization; United Nations Children's Fund. Iron deficiency anemia: assessment, prevention, and control: a guide for programme managers. Geneva: World Health Organization; 2001.

6. Departamento de Atenção Básica, Secretaria de Atenção à Saúde, Ministério da Saúde. NutriSUS: guia de evidências: estratégia de fortificação da alimentação infantil com micronutrientes (vitaminas e minerais) em pó. Brasília: Ministério da Saúde; 2015.

7. Ministério da Saúde. NutriSUS: caderno de orientações: estratégia de fortificação da alimentação infantil com micronutrientes (vitaminas e minerais) em pó. Brasília: Ministério da Saúde/Ministério da Educação; 2015.

8. Ministério da Saúde. Suplemento alimentar com múltiplos micronutrientes em pó para implantação do NutriSUS. Relatório de recomendação da Comissão Nacional de Incorporação de Tecnologias no SUS - CONITEC. Brasília: Ministério da Saúde; 2014. 
9. Batista Filho M, Ferreira LOC. Prevenção e tratamento da anemia nutricional ferropriva: novos enfoques e perspectivas. Cad Saúde Pública 1996; 12:411-5.

10. Secretaria de Atenção à Saúde, Ministério da Saúde. Estratégia Nacional para Promoção do Aleitamento Materno e Alimentação Complementar Saudável no Sistema Único de Saúde: manual de implementação. Brasília: Ministério da Saúde; 2015.

11. Departamento de Atenção Básica, Secretaria de Atenção à Saúde, Ministério da Saúde. Programa Nacional de Suplementação de Ferro: manual de condutas gerais. Brasília: Ministério da Saúde; 2013.

12. Agência Nacional de Vigilância Sanitária. Resolução no 344, de 13 de dezembro de 2002. Regulamento técnico para a fortificação das farinhas de trigo e farinhas de milho com ferro e ácido fólico. Diário Oficial da União 2012; 18 dez.

13. Brasil. Decreto no 6.286, de 5 de dezembro de 2007. Institui o Programa Saúde na Escola PSE, e dá outras providências. Diário Oficial da União 2007; 5 dez.

14. Dias PC, Henriques P, Ferreira DM, Barbosa RMS, Soares DSB, Luquez TMS, et al. Desafios da intersetorialidade nas políticas públicas: o dilema entre a suplementação nutricional e a promoção da alimentação saudável em escolas. Cad Saúde Pública 2018; 34:e00035218.

15. Faria CAP. Ideias, conhecimento e políticas públicas: um inventário sucinto das principais vertentes analíticas recentes. Rev Bras Ciênc Soc 2003; 18:21-9.

16. Muller P. As políticas públicas. Niterói: Eduff; 2008.

17. Lima LL, D’Ascenzi L. Implementação de políticas públicas: perspectivas analíticas. Revista de Sociologia e Política 2013; 21:101-10.

18. Frey K. Políticas públicas: um debate conceitual e reflexões referentes à prática da análise de políticas públicas no Brasil. Planej Polít Públicas 2000; 21:211-59.

19. Hall PA. The role of interests, institutions and ideas in the comparative political economy of the industrialized nations. In: Lichbach MI, Zuckerman AS, editors. Comparative politics: rationality, culture, and structure. Cambridge: Cambridge University Press; 1997. p. 174-207.

20. Ministério da Saúde. Portaria MS/SVS no 31, de 13 de janeiro de 1998. Dispõe regulamento técnico para fixação de identidade e qualidade de alimentos adicionados de nutrientes essenciais. Diário Oficial da União 1998; 16 jan.

21. Ministério da Saúde. Estratégia de fortificação caseira no Brasil: workshop 29 e 30 de setembro de 2011. Brasília: Ministério da Saúde; 2012.

22. Organização Mundial da Saúde. Diretriz: uso de formulações em pó de múltiplos micronutrientes para fortificação caseira de alimentos consumidos por bebês e crianças de 6-23 meses de vida. Genebra: Organização Mundial da Saúde; 2013.
23. Home Fortification Technical Advisory Group. Programatic guidance brief on use of micronutrient powder (MNP) for home fortification. s.l.: Home Fortification Technical Advisory Group; 2011.

24. Brasil. Lei no 11.947, de 16 de junho de 2009. Dispõe sobre o atendimento da alimentação escolar e do Programa Dinheiro Direto na Escola aos alunos da educação básica. Diário Oficial da União 2009; 16 jun.

25. Fundo Nacional de Desenvolvimento da Educação. Resolução no 6, de 8 de maio de 2020. Dispõe sobre o atendimento da alimentação escolar aos alunos da educação básica no âmbito do Programa Nacional de Alimentação Escolar - PNAE. Diário Oficial da União 2020; 8 mai.

26. Brasil. Decreto no 7.272 , de 25 de agosto de 2010. Regulamenta a Lei no 11.346 , de 15 de setembro de 2006 e institui a Política Nacional de Segurança Alimentar e Nutricional - PNSAN. Diário Oficial da União 2010; 26 ago.

27. Iglesias A, Dalbello-Araujo M. As concepções de promoção da saúde e suas implicações. Cad Saúde Colet (Rio J.) 2011; 19:291-8.

28. Bezerra IMP, Sorpreso ICE. Conceitos de saúde e movimentos de promoção da saúde em busca da reorientação de práticas. J Hum Growth Dev 2016; 26:11-6.

29. Moraes MCL. Promoção da saúde: visitando conceitos e ideias. Revista Família, Ciclo de Vida e Saúde no Contexto Social 2017; 5:75-9.

30. Furtado M, Szapiro A. Promoção da saúde e seu alcance biopolítico: o discurso sanitário da sociedade contemporânea. Saúde Soc 2012; 21:811-21

31. Westphal MF. Promoção da saúde e prevenção de doenças. In: Campos GWS, Minayo MCS, Akerman M, Drumond Jr. M, Carvalho YM, organizadores. Tratado de saúde coletiva. São Paulo: Hucitec Editora/Rio de Janeiro: Editora Fiocruz; 2006. p. 635-67.

32. Ministério da Saúde. Relação Nacional de Medicamentos Essenciais: Rename 2020. https:// bvsms.saude.gov.br/bvs/publicacoes/relacao_ medicamentos_rename_2020.pdf (acessado em 16/Out/2020).

33. Ministério da Saúde. Balanço de gestão 20152018 - Coordenação Geral de Alimentação e Nutrição (CGAN). Brasília: Ministério da Saúde; 2018.

34. Ministério da Saúde. Portaria MS/SVS no 32, de 13 de janeiro de 1998. Regulamento técnico para fixação de identidade e qualidade de suplementos vitamínicos e ou de minerais. Diário Oficial da União 1998; 15 jan.

35. Agência Nacional de Vigilância Sanitária. Resolução no 269, de 22 de setembro de 2005 . Regulamento técnico sobre a ingestão diária recomendada (IDR) de proteína, vitaminas e minerais. Diário Oficial da União 2005; 23 set.

36. Food and Nutrition Board, Institute of Medicine. Dietary reference intakes. Washington DC: National Academic Press; 2001. 
37. Food and Agriculture Organization of the United Nations; World Health Organization. Human vitamin and mineral requirements. Report of a joint FAO/WHO expert consultation. Bangkok: Food and Agriculture Organization of the United Nations/World Health Organization; 2001.

38. Sociedade Brasileira de Pediatria. Anemia ferropriva em lactentes: revisão com foco na prevenção. São Paulo: Departamento Científico de Nutrologia, Sociedade Brasileira de Pediatria; 2012.

39. Schauer C, Zlotkin S. Home fortification with micronutrient sprinkles: a new approach for the prevention and treatment of nutritional anemias. Paediatr Child Health 2003; 8:87-90.

40. Nguyen M, Poonawala A, Leyvraz M, Berger J, Schofield D, Nga TT, et al. A delivery model for home fortification of complementary foods with micronutrient powders: innovation in the context of Vietnamese health system strengthening. Nutrients 2016; 8:259.

41. Creed-Kanashiro H, Bartolini R, Abad M, Arevalo V. Promoting multi-micronutrient powders (MNP) in Peru: acceptance by caregivers and role of health personnel. Matern Child Nutr 2016; 12:152-63.

42. Leyvraz M, David-Kigaru DM, MachariaMutie C, Aaron GJ, Roefs M, Tumilowicz A. Coverage and consumption of micronutrient powders, fortified staples, and iodized salt among children aged 6 to 23 months in selected neighborhoods of Nairobi County, Kenya. Food Nutr Bull 2018; 39:107-15.

43. Korenromp EL, Adeosun O, Adegoke F, Akerele A, Anger C, Ohajinwa C, et al. Micronutrient powder distribution through Maternal, Neonatal and Child Health Weeks in Nigeria: process evaluation of feasibility and use. Public Health Nutr 2015; 19:1882-92.

44. De-Regil LM, Suchdev PS, Vist GE, Walleser S, Peña-Rosas JP. Home fortification of foods with multiple micronutrient powders for health and nutrition in children under two years of age. Cochrane Database Syst Rev 2011; (9):CD008959.

45. Bhutta ZA, Ahmed T, Black RE, Cousens S, Dewey K, Giugliani E, et al. What works? Interventions for maternal and child undernutrition and survival. Lancet 2008; 371:417-40.

46. Engle PL. Strategies for reducing inequalities and improving developmental outcomes for young children in low-income and middleincome countries. Lancet 2011; 378:1339-53.

47. Dewey KG, Yang Z, Boy E. Systematic review and meta-analysis of home fortification of complementary foods. Matern Child Nutr 2009; 5:283-321.

48. Munayco CV, Ulloa-Rea ME, Medina-Osis J, Lozano-Revollar CR, Tejada V, Castro-Salazar $\mathrm{C}$, et al. Evaluación del impacto de los multimicronutrientes en polvo sobre la anemia infantil en tres regiones andinas del Perú. Rev Peru Med Exp Salud Pública 2013; 30:229-34.
49. Ministério da Saúde. Guia alimentar para a população brasileira: promovendo a alimentação saudável. Brasília: Ministério da Saúde; 2006.

50. Ministério da Saúde. Escolas promotoras de saúde: experiências do Brasil. Brasília: Ministério da Saúde; 2006. (Série Promoção da Saúde, 6).

51. Ministério da Saúde. Dez passos para uma alimentação saudável: guia alimentar para crianças menores de dois anos: um guia para o profissional da saúde na atenção básica. $2^{\text {a }} \mathrm{Ed}$. Brasília: Ministério da Saúde; 2013.

52. Departamento de Atenção Básica, Secretaria de Atenção à Saúde, Ministério da Saúde. Guia alimentar para a população brasileira. 2a Ed. Brasília: Ministério da Saúde; 2014.

53. Ministério da Saúde. Guia alimentar para crianças brasileiras menores de 2 anos. Brasília: Ministério da Saúde; 2019.

54. Institute of Medicine. Dietary reference intakes for vitamina $\mathrm{A}$, vitamina $\mathrm{K}$, arsenic, boron, chrominum, copper, iodine, iron, manganese, molybdenum, nickel, silicon, vanadium and zinc. Washington DC: National Academic Press; 2001.

55. Ministério da Educação. Resolução/FNDE/ MEC no 26, de 17 de junho de 2013. Dispõe sobre o atendimento da alimentação escolar aos alunos da educação básica no âmbito do programa nacional de alimentação escolar PNAE. Diário Oficial da União 2013; 17 jun.

56. Burlandy L, Alexandre VP, Gomes FS, Castro IRR, Dias PC, Henriques P, et al. Políticas de promoção de saúde e potenciais conflitos de interesses que envolvem o setor privado comercial. Ciênc Saúde Colet 2016; 21:1809-18.

57. Burlandy L, Gomes FS, Carvalho CMP, Dias PC, Henriques P. Intersetorialidade e potenciais conflitos de interesse entre governo e setor privado comercial no âmbito das ações de alimentação e nutrição para o enfrentamento de doenças crônicas não transmissíveis. Vigil Sanit Debate 2014; 2:124-9.

58. Melo MFG, Santos LMP, Lira PIC. Uso de suplementos vitamínicos e/ou minerais por crianças menores de seis meses no interior do estado de Pernambuco. Rev Bras Saúde Mater Infant 2005; 3:359-66.

59. Ministério da Saúde. Portaria MS/SVS no 40, de 13 de janeiro de 1998. Regulamento que estabelece normas para níveis de dosagens diários de vitaminas e minerais em medicamentos. Diário Oficial da União 1998; 16 jan. 


\section{Abstract}

Iron deficiency anemia affects different populations worldwide, and in Brazil it is one of the principal childhood nutritional deficiencies. Different strategies have been proposed by international agencies and the Brazilian Ministry of Health for its prevention. The study aimed to analyze the ideas in dispute, expressed in official documents and narratives by health and education workers concerning the use of nutritional supplementation and/or fortification as a measure in schools to prevent anemia. The study was based on government documents and semi-structured interviews with workers in municipalities that implemented the school food fortification strategy (NutriSUS) in the state of Rio de Janeiro. The ideas expressed in the government documents highlight the efficiency of home supplementation and fortification for prevention of anemia, based on scientific studies and adjusted to the objectives of the public policies set out in them. Some ideas in the workers' narratives are critical of the need for this type of intervention, others they favor them, indicating controversies in the process of local operationalization of federal policies. Tensions were observed in the concepts of promotion, prevention, and treatment through NutriSUS, dosage, and form of administration. Criticisms of supplementation highlight healthy eating practices as the best strategy. Medicalization rather than health promotion measures can strain the pedagogical perspective in schools and produce contradictory ideas on the best strategies for the promotion of healthy eating.

\section{Health Public Policy; Anemia; School Feeding; Dietary Supplements}

\section{Resumen}

La anemia por deficiencia de hierro afecta mundialmente a diferentes poblaciones y, en Brasil, es una de las principales carencias nutricionales $d u$ rante la infancia. Se proponen diferentes estrategias por parte de organismos internacionales y el Ministerio de Salud para su prevención. El objetivo del estudio fue analizar las ideas en disputa, presentes en documentos oficiales y narraciones de los profesionales de salud y educación, acerca del uso de los suplementos y/o enriquecimientos nutricionales, como medida de prevención de la anemia en el ámbito escolar. Se basó en el análisis de documentos gubernamentales y entrevistas semiestructuradas con profesionales que actuaban en municipios que implementaron la estrategia de fortificación de la alimentación escolar (NutriSUS) en el estado de Río de Janeiro. Las ideas difundidas en los documentos gubernamentales resaltan la eficiencia de la suplementación y del enriquecimiento alimenticio casero para la prevención de anemia, basadas investigaciones científicas, y se adecua a los objetivos de las politicas públicas en ellos formalizados. Las ideas presentes en las narraciones de los profesionales son, a veces, críticas con la necesidad de este tipo de intervención y, otras veces, favorables, indicando las controversias presentes en el propio proceso de operacionalización local de las políticas federales. Se observaron tensiones en torno a los conceptos de promoción, prevención y tratamiento mediante NutriSUS, así como sobre la dosificación y la forma de administración. Las críticas a los suplementos resaltan las prácticas alimentarias saludables como estrategia preferente. La medicalización en detrimento de acciones de promoción de la salud puede tensionar la perspectiva pedagógica en el contexto escolar y producir ideas contradictorias sobre las mejores estrategias de promoción de alimentación saludable.

Politica Pública de Salud; Anemia; Alimentación Escolar; Suplementos Dietéticos
Recebido em 04/Jan/2021

Versão final reapresentada em 29/Jul/2021

Aprovado em 13/Ago/2021 